\title{
HÁBITOS DE ATIVIDADE FÍSICA E LAZER DE ADOLESCENTES
}

\author{
Thiago Sousa Matias \\ Universidade do Estado de Santa Catarina, Florianópolis, Santa Catarina, Brasil \\ Martina Kieling Sebold Barros Rolim \\ Universidade do Estado de Santa Catarina, Florianópolis, Santa Catarina, Brasil \\ Camilie Pacheco Schmoelz \\ Universidade do Estado de Santa Catarina, Florianópolis, Santa Catarina, Brasil
}

\author{
Alexandro Andrade \\ Universidade do Estado de Santa Catarina, Florianópolis, Santa Catarina, Brasil
}

\begin{abstract}
Resumo
Este estudo analisou os hábitos de atividade física e lazer de 216 adolescentes estudantes de um centro federal de educação tecnológica de Santa Catarina. Os adolescentes foram avaliados através do Questionário de Atividades Físicas Habituais e do Questionário de Autoavaliação do Estilo de Vida. Os dados foram tratados com estatística descritiva e inferencial, teste " $t$ " de Student e Qui-quadrado. As atividades físico-esportivas preferidas pelos adolescentes no lazer são os esportes coletivos $(33,0 \%)$. Meninos preferem mais o futebol (44\%), e meninas, a caminhada (35,7\%). Mais da metade das atividades de lazer $(56,8 \%)$ é de caráter sedentário, entretanto, os adolescentes ativos optam mais pelo lazer ativo que os sedentários $(\mathrm{p}=0,000)$.
\end{abstract}

Palavras-chave: Atividade Motora. Atividades de Lazer. Adolescente.

\section{Introdução}

— studos atuais têm demonstrado que a prática de atividade física contribui para a saúde e o bem-estar da população em geral, estando associada a uma série de benefícios físicos, psicológicos e sociais (BOICHÉ e SARRAZIN, 2007; CDC, 2006; 2008; OMS, 2005;).

De acordo com o Center for Disease Control and Prevention (CDC), na adolescência, os benefícios da atividade física já podem ser alcançados em sessões acumuladas de atividades moderadas de cerca de dez minutos. Sendo assim, a atividade física necessária para a obtenção de benefícios à saúde pode ser escolhida e realizada no tempo de lazer dos adolescentes (BIDDLES e MUTRIE, 2001). 
Entretanto, as atividades físicas praticadas no tempo destinado ao lazer representam um domínio da vida cotidiana organizado segundo determinadas convenções como as biológicas, psicológicas e sociais (SALLES-COSTA et al., 2003), sendo muitas vezes colocadas em competição com outros valores, comportamentos e atitudes, que podem ter mais relevância para os desejos dos jovens (SANTOS et al., 2005).

Entendendo que na adolescência se formam valores e atitudes para a adoção de um estilo de vida saudável, (ROLIM, 2007; ALVES et al., 2005; PERKINS et al., 2004), torna-se importante conhecer os hábitos de adolescentes frente à prática de atividade física no lazer para influenciar de maneira decisiva o comportamento dos jovens na apropriação de um estilo de vida saudável (SANTOS et al., 2005). Sendo assim, e considerando o exposto, o objetivo deste estudo foi analisar os hábitos de atividade física e lazer de adolescentes estudantes de um centro federal de educação tecnológica de Santa Catarina.

\section{Método}

Trata-se de um estudo de campo, de natureza descritiva, autorizado pelo Comitê de Ética em Pesquisa em Seres Humanos (CEP), da Universidade do Estado de Santa Catarina (número de referência 018/06).

A população deste estudo constitui-se de adolescentes de 13 a 19 anos, estudantes do ensino médio de um centro federal de educação tecnológica de Santa Catarina, Brasil. A constituição da amostra obedeceu à participação voluntária de 216 adolescentes, divididos por sexo e nível habitual de atividade física (ativos e sedentários). Foi solicitado aos alunos a assinatura do Termo de Consentimento Livre e Esclarecido para participação na pesquisa; e aos menores de 18 anos, foi exigida também a assinatura dos pais.

Instrumentos

Para avaliação do nível habitual de atividade física, utilizou-se o Questionário de Atividades Físicas Habituais - QAFH, desenvolvido por Pate e adaptado por Nahas (2001) para adolescentes brasileiros. A pontuação obtida no QAFH permite classificar os adolescentes em quatro grupos: de 0 a 5 pontos $=$ inativo; de 6 a 11 pontos $=$ moderadamente ativo; de 12 a 20 pontos $=$ ativo e com 21 pontos ou mais $=$ 
muito ativo. Para este estudo, em função de ser um estudo piloto, a classificação foi feita nomeando os indivíduos que pontuarem entre 0 e 5 pontos como "sedentários", acima de 12 pontos de "ativos" e excluindo da amostra os adolescentes moderadamente ativos (6 e 11 pontos).

Os dados referentes aos hábitos de atividade física e lazer dos adolescentes foram retirados do Questionário de Autoavaliação do Estilo de Vida (ROLIM, 2007) adaptado de Andrade (2001). Trata-se de um questionário misto, com questões abertas e fechadas, composto por uma caracterização geral e quatro itens: (a) indicadores de saúde; (b) hábitos de atividade física e lazer; (c) ambiente de convívio e (d) fé e espiritualidade. Assim como em estudos anteriores (MATIAS et al., 2009, 2010; ROLIM et al., 2007), foi utilizado apenas o item referente aos hábitos de atividades físicas e lazer.

Procedimentos da coleta de dados

Em relação à coleta de dados com os alunos, esta foi realizada após a entrega e o recolhimento dos Termos de Consentimento Livre Esclarecido dos participantes da pesquisa (alunos e pais dos alunos menores de 18 anos). O esclarecimento quanto aos procedimentos de preenchimento do questionário foi realizado minutos antes da coleta, em horário letivo, na própria sala de aula, sempre na presença de pesquisadores treinados da equipe do Laboratório de Psicologia do Esporte e do Exercício - LAPE, da Universidade do Estado de Santa Catarina - UDESC.

Os dados foram tabulados e após os procedimentos de categorização das variáveis, as informações foram registradas em um banco de dados para a efetivação das análises estatísticas, utilizando o software Statistical Package for the Social Science (SPSS) para Windows, versão 17.0.

O tratamento dos dados foi realizado em duas etapas. Na primeira, foi realizada estatística descritiva e os testes de normalidade (Kolmogorov-Smirnov). Na segunda etapa foi aplicada estatística inferencial. Para realizar as comparações entre os grupos foi utilizado o teste " $\mathrm{t}$ " de student para amostras independentes e o teste "Qui-quadrado" para observar associação entre as variáveis nominais. O nível de significância estabelecido foi de $\alpha=0,05$. 


\section{Resultados}

Caracterização e atividades de lazer

A média de idade dos adolescentes é de 16,2 $\pm 1,1$ anos, sendo de $16,3 \pm 1,1$ anos para os meninos e $16 \pm 1,1$ anos para as meninas. A maioria da amostra $(61,1 \%)$ é constituída de meninos e $58,3 \%$ dos adolescentes são ativos. Observou-se que os meninos $(68,2 \%)$ são significativamente mais ativos que as meninas $(44,0 \%)(\mathrm{p}=0,001)$.

A atividade de lazer preferida pelos adolescentes, independente do nível de atividade física, é, em primeiro lugar, o uso do computador $(29,2 \%)$, seguido pelas atividades físico-esportivas $(29,6 \%)$ (Tabela $1)$.

Tabela 1: Atividades de lazer preferidas pelos adolescentes sedentários e ativos

\begin{tabular}{lcccccc}
\hline Atividades de lazer preferidas & \multicolumn{2}{c}{ Total } & \multicolumn{2}{c}{ Sedentários } & \multicolumn{2}{c}{ Ativos } \\
\cline { 2 - 7 } & $f$ & $\%$ & $f$ & $\%$ & $F$ & $\%$ \\
\hline Computador & 63 & $\mathbf{2 9 , 2}$ & 29 & 32,6 & 34 & 26,8 \\
Atividades físico-esportivas & 64 & $\mathbf{2 9 , 6}$ & 6 & 6,7 & 58 & 45,7 \\
Leitura & 14 & 6,5 & 11 & 12,4 & 3 & 2,4 \\
Assistir Televisão & 12 & 5,6 & 9 & 10,1 & 3 & 2,4 \\
Tocar instrumento & 9 & 4,2 & 2 & 2,2 & 7 & 5,5 \\
Outras Atividades & 51 & 23,6 & 30 & 33,7 & 21 & 16,5 \\
Não respondeu & 3 & 1,4 & 2 & 2,2 & 1 & 0,8 \\
Total & 216 & 100 & 89 & 100,0 & 127 & 100,0 \\
\hline
\end{tabular}

Quanto às características das atividades de lazer, os adolescentes sedentários preferem as atividades de lazer que possuem pouca ou nenhuma interação social, nestes casos, atividades sem gasto energético. Nos mais citados estão uso do computador, leitura e assistir televisão. Os adolescentes ativos, por sua vez, têm preferência por atividades de lazer relacionadas às atividades de caráter esportivo, mesmo optando também por atividades de caráter sedentário. As atividades de lazer estão associadas ao nível habitual de atividade física dos adolescentes $(\mathrm{p}=0,000)$.

Considerando todas as atividades de lazer citadas pelos adolescentes, mais da metade $(69,1 \%)$ é de caráter sedentário, sem distinção entre sexo ou nível habitual de atividade física. A escolha por atividades sedentárias corresponde à 53,5\% entre os indivíduos ativos e 91,1\% entre os indivíduos sedentários. Embora os adolescentes, de maneira geral, optem mais por atividades de lazer sedentárias, os adolescentes ativos optam mais pelo lazer ativo $(45,7 \%)$ do que adolescentes seden- 
tários $(6,7 \%)$, tanto os meninos quanto as meninas $(\mathrm{p}=0,000 ; \mathrm{p}=0,000)$.

Ao analisar as atividades referidas pelos adolescentes separadas por sexo, observa-se que a escolha por atividades sedentárias corresponde a $65,2 \%$ entre os meninos e $75,0 \%$ entre as meninas. Ressalta-se também que os meninos optam mais por atividades de lazer ativo (33,3\%) do que as meninas $(23,8 \%)$ (Tabela 2 ) reforçando os dados acerca do nível de atividade física.

Tabela 2: Atividades de lazer preferidas por meninos e meninas

\begin{tabular}{lcccccc}
\hline Atividades de lazer preferidas & \multicolumn{2}{c}{ Total } & \multicolumn{2}{c}{ Meninos } & \multicolumn{2}{c}{ Meninas } \\
\cline { 2 - 7 } & $f$ & $\%$ & $f$ & $\%$ & $F$ & $\%$ \\
\hline Computador & 63 & $\mathbf{2 9 , 2}$ & 43 & $\mathbf{3 2 , 6}$ & 20 & $\mathbf{2 3 , 8}$ \\
Atividades físico-esportivas & 64 & $\mathbf{2 9 , 6}$ & 44 & $\mathbf{3 3 , 3}$ & 20 & $\mathbf{2 3 , 8}$ \\
Leitura & 14 & 6,5 & 9 & 6,8 & 5 & 6,0 \\
Assistir Televisão & 12 & 5,6 & 4 & 3,0 & 8 & 9,5 \\
Tocar instrumento & 9 & 4,2 & 7 & 5,3 & 2 & 2,4 \\
Outras Atividades & 51 & 23,6 & 23 & 17,4 & 28 & 33,3 \\
Não respondeu & 3 & 1,4 & 2 & 1,5 & 1 & 1,2 \\
Total & 216 & 100 & 132 & 100 & 84 & 100 \\
\hline
\end{tabular}

Atividades físico-esportivas no lazer

As atividades físico-esportivas mais praticadas pelos adolescentes no lazer são os esportes coletivos, sendo que o futebol corresponde à $11,11 \%$ de todas as atividades. Na sequência, as atividades de lazer dos adolescentes estão distribuídas em atividades aeróbicas, lutas, danças, entre outras. Obviamente, a maior parte destas atividades é realizada por adolescentes ativos, enquanto a grande maioria $(91,01 \%)$ dos adolescentes sedentários não opta por atividades físico-esportivas nos momentos de lazer (Tabela 3). 
Tabela 3: Atividades físico-esportivas praticadas pelos adolescentes ativos e sedentários

\begin{tabular}{lllllll}
\hline Atividades Físico- & \multicolumn{7}{l}{ Total } & \multicolumn{2}{l}{ Sedentários } & \multicolumn{2}{l}{ Ativos } \\
\cline { 2 - 7 } Esportivas & $f$ & $\%$ & $F$ & $\%$ & $f$ & $\%$ \\
\hline Basquetebol & 3 & 1,39 & -- & - & 3 & 2,36 \\
Boliche & 1 & 0,46 & 1 & 1,12 & -- & -- \\
Caminhar & 5 & 2,31 & - & -- & 5 & 3,94 \\
Dançar & 5 & 2,31 & 2 & 2,25 & 3 & 2,36 \\
Educação fisica & 1 & 0,46 & -- & -- & 1 & 0,79 \\
Esportes & 1 & 0,46 & -- & -- & 1 & 0,79 \\
Futebol & $\mathbf{2 4}$ & $\mathbf{1 1 , 1 1}$ & 1 & 1,12 & $\mathbf{2 3}$ & $\mathbf{1 8 , 1 1}$ \\
Handebol & 1 & 0,46 & -- & -- & 1 & 0,79 \\
Jump & 1 & 0,46 & -- & - & 1 & 0,79 \\
Karatê & 1 & 0,46 & -- & -- & 1 & 0,79 \\
Kung-fu & 2 & 0,93 & -- & -- & 2 & 1,57 \\
Mergulhar & 1 & 0,46 & -- & -- & 1 & 0,79 \\
Musculação & 3 & 1,39 & -- & -- & 3 & 2,36 \\
Pedalar & 3 & 1,39 & -- & -- & 3 & 2,36 \\
Pescar & 1 & 0,46 & -- & -- & 1 & 0,79 \\
Surfe & 1 & 0,46 & -- & -- & 1 & 0,79 \\
Tênis & 1 & 0,46 & -- & - & 1 & 0,79 \\
Voleibol & 9 & 4,17 & 2 & 2,25 & 7 & 5,51 \\
Nenhuma & 149 & 68,98 & $\mathbf{8 1}$ & $\mathbf{9 1 , 0 1}$ & 68 & 53,54 \\
Não respondeu & 3 & 1,39 & 2 & 2,25 & 1 & 0,79 \\
Total & 216 & 100 & 89 & 100 & 127 & 100 \\
\hline
\end{tabular}

Quando separados por sexo, observa-se que os meninos preferem o futebol $(15,91 \%)$ e as meninas a dança $(5,95 \%)$ e o voleibol $(5,95 \%)$. Reforçando os dados sobre o nível de atividade física, a maior parte das meninas não opta por atividades físico-esportivas nos momentos de lazer (75\%). (Tabela 4). 
Tabela 4: Atividades físico-esportivas praticadas por meninos e meninas

\begin{tabular}{|c|c|c|c|c|c|c|}
\hline \multirow{2}{*}{ Atividades Físico-Esportivas } & \multicolumn{2}{|c|}{ Total } & \multicolumn{2}{|c|}{ Meninos } & \multicolumn{2}{|c|}{ Meninas } \\
\hline & $f$ & $\%$ & $\bar{f}$ & $\%$ & $\bar{f}$ & $\%$ \\
\hline Basquetebol & 3 & 1,39 & 3 & 2,27 & -- & -- \\
\hline Boliche & 1 & 0,46 & & & 1 & 1,19 \\
\hline Caminhar & 5 & 2,31 & 1 & 0,76 & 4 & 4,76 \\
\hline Dançar & 5 & 2,31 & & & 5 & 5,95 \\
\hline Educação física & 1 & 0,46 & 1 & 0,76 & -- & - \\
\hline Esportes & 1 & 0,46 & 1 & 0,76 & -- & -- \\
\hline Futebol & 24 & 11,11 & 21 & 15,91 & 3 & 3,57 \\
\hline Handebol & 1 & 0,46 & & & 1 & 1,19 \\
\hline Jump & 1 & 0,46 & 1 & 0,76 & -- & -- \\
\hline Karatê & 1 & 0,46 & 1 & 0,76 & -- & -- \\
\hline Kung-fu & 2 & 0,93 & 2 & 1,52 & -- & -- \\
\hline Mergulhar & 1 & 0,46 & & & 1 & 1,19 \\
\hline Musculação & 3 & 1,39 & 3 & 2,27 & -- & -- \\
\hline Pedalar & 3 & 1,39 & 3 & 2,27 & -- & -- \\
\hline Pescar & 1 & 0,46 & 1 & 0,76 & -- & -- \\
\hline Surfe & 1 & 0,46 & 1 & 0,76 & -- & -- \\
\hline Tênis & 1 & 0,46 & 1 & 0,76 & -- & -- \\
\hline Voleibol & 9 & 4,17 & 4 & 3,03 & 5 & 5,95 \\
\hline Nenhuma & 149 & 68,98 & 89 & 67,42 & 63 & 75,00 \\
\hline Não respondeu & 3 & 1,39 & 2 & 1,52 & 1 & 1,19 \\
\hline Total & 216 & 100 & 132 & 100 & 84 & 100 \\
\hline
\end{tabular}

\section{Discussão dos resultados}

Torna-se difícil comparar os resultados em relação ao nível habitual de atividade física neste estudo com outras pesquisas brasileiras, ao passo que os resultados variam de $22,5 \%$ de sedentarismo para adolescentes do sexo masculino de Pelotas/RS (OEHLSCHLAEGER et al., 2004) a 94\% em adolescentes do sexo feminino de Niterói/RJ (SILVA, MALINA, 2000). Segundo Tassitano et al (2007) essas variações não ocorrem apenas por diferenças regionais, mas são também muito influenciadas pelos diferentes parâmetros para a avaliação do nível de atividade física. O que se observa é que, independente da instrumentalização utilizada, a maior parte dos estudos indica alto índice de sedentarismo entre os adolescentes brasileiros (SILVA, MALINA, 2000; TASSITANO et al., 2007; VASQUES e LOPES, 2009).

Embora os resultados deste estudo apontem que mais da metade dos adolescentes são fisicamente ativos, se observa um grande número de adolescentes que não praticam nenhum tipo de atividade física. A preocupação se agrava ao analisar estudos na mesma região que avaliam a atitude dos adolescentes frente à atividade física em que se cons- 
tata que um grande número de adolescentes não pratica e não pretende iniciar a prática de atividade física (VIANA; ANDRADE e MATIAS, 2010; MATIAS et al., 2009, 2010; ROLIM et al., 2007).

Matias et al. (2010) destacam que há uma diminuição do nível de atividade física durante o período da adolescência que pode ser explicada, tanto pelas mudanças fisiológicas que ocorrem durante este período, quanto pelo aumento do número de atividades ocupacionais dos adolescentes referentes à entrada no Ensino Médio e no mercado de trabalho.

A proporção do número de meninos ativos em relação às meninas ativas, independente do instrumento utilizado, é um fenômeno comum encontrado na literatura (GOMES, SIQUEIRA e SICHIERI, 2001; MATSUDO et al., 2002; PIRES et al. 2004; DE-BOURDEAUDHUIJ et al., 2004; OEHLSCHLAEGER et al., 2004; ALVES et al., 2005; MASCARENHAS, et al., 2005; REIS e PETROSKI, 2005; SOUZA e DUARTE, 2005; TELAMA, NUPPONEN e PIERÓN, 2005; GUEDES et al., 2006; LI et al., 2007) e assim parece que o sexo masculino é um fator positivamente associado a um maior nível de atividade física.

Esse fenômeno pode ser justificado pelas diferenças no âmbito sóciocultural e comportamental dos adolescentes. Conti et al (2005) afirmam, por exemplo, que desde a infância os meninos são estimulados às práticas esportivas e às atividades físicas como subir em árvores, andar de bicicleta, e, principalmente "jogar bola", enquanto as meninas são mais encorajadas ao desenvolvimento de atividades tipicamente sedentárias. Outros estudos referem-se à busca da identidade sexual (SEABRA et al., 2008), à percepção de baixa auto-eficácia para a prática esportiva (BIDDLE et al., 2006), ao desconforto provocado por dores e sudoreses e à aversão a competições (GRIESER et al., 2006) como alguns empecilhos.

Contudo, há que se refletir o caráter da magnitude das diferenças encontradas entre meninos e meninas, assim, mais do que reforçar um paradigma entre sexo, é necessário questionar até que ponto as condições socioambientais são as mesmas para que meninos e meninas pratiquem atividade física (MATIAS et al., 2010).

Quanto às atividades de lazer, as de caráter sedentário são preferidas tanto pelos adolescentes sedentários quanto pelos ativos. Isto indica que hábitos sedentários não interferem no envolvimento dos adolescentes em atividades físicas e estão de acordo com outros estu- 
dos nos quais as atividades de lazer sedentárias aparecem como as preferidas dos adolescentes (MATSUDO et al.,2002; SARRIERA et al., 2007; PFEIFER; MARTINS; SANTOS, 2010).

Outros estudos (TELAMA, NUPPONEN e PIERÓN 2005; NOBRE, 2006) também encontraram relação positiva e significativa entre hábitos de lazer sedentários e adolescentes ativos. Todavia, é importante observar que os comportamentos ativos são preferidos pelos adolescentes ativos e os jovens podem se interessar tanto por atividades fisicamente ativas quanto por sedentárias.

O problema existe quando os adolescentes optam somente pelas atividades sedentárias e sem envolvimento social. Neste caso, a literatura tem apontado que as atividades de caráter sedentário mostram-se constantes na população de adolescentes (PETER et al., 2008). Um estudo recente com adolescentes portugueses mostrou que as atividades com características sedentárias predominam, das vinte e uma atividades listadas apenas duas são necessariamente de caráter ativo, e estas, na ordem de preferências, aparecem na décima quarta e décima quinta posição (ESCULCAS e MOTA, 2005).

Com relação à prática de diferentes atividades físico-esportivas nos momentos de lazer pelos adolescentes investigados, foi observado que as preferências por determinadas modalidades e as diferenças entre sexos na adesão destas modalidades, como o futebol entre os meninos e a caminhada entre as meninas, refletem as manifestações culturais que se desenvolvem no país. O futebol é o esporte de preferência nacional e o mais divulgado pela mídia e a caminhada representa uma atividade de baixo impacto e baixo desconforto. O futebol como atividade preferida entre os meninos e a caminhada entre as meninas está de acordo com outros estudos realizados com adolescentes brasileiros (NOBRE, 2006; SILVA e MALINA, 2000).

Salles-Costa et al. (2003) consideram ainda que as atividades físicas praticadas no tempo destinado ao lazer representam um domínio da vida cotidiana, organizado segundo determinadas convenções. Entre elas as concepções acerca do ideal de corpo segundo o sexo, onde homens e mulheres apresentam comportamentos distintos no que se refere à prática de exercícios físicos. Assim, percebe-se que a cultura esportiva do país, fortemente influenciada pela mídia, determina a principal atividade praticada pelos meninos (futebol) e as questões biológicas determinam as atividades preferidas pelas meninas (caminhada). 


\title{
Conclusões
}

Com relação às opções de lazer, a preferência dos jovens é prioritariamente sedentária, entretanto os adolescentes ativos optam mais pelo lazer ativo do que os sedentários. Estes dados são alarmantes ao observar em boa parte da amostra comportamentos de lazer restritos às atividades sedentárias. Assim destaca-se a importância do equilíbrio nas escolhas das atividades de lazer e a valorização de diversas competências como o esporte, exercícios físicos, música, leitura, dança, entre outras.

Por fim, ao analisar as atividades físico-desportivas observa-se a crença dos esportes coletivos e a diferença entre sexo na escolha das atividades. Estas características devem ser levadas em conta no desenvolvimento de programas de atividades físico-esportivas para jovens. Entretanto há que se oportunizar, nessa fase da vida, a experimentação de diversas atividades, sobretudo em relação ao sexo, em que meninos e meninas são estigmatizados em determinadas atividades.

\section{Adolescents' habits of physical activity and leisure}

\begin{abstract}
This study aimed to investigate the physical activities and leisure habits of 216 adolescents from a federal center of technological education from Santa Catarina. Atividades Físicas Habituais questionnaire and Autoavaliação do Estilo de Vida questionnaire were used. Data were analyzed by descriptive and inferential statistics, "t" test and Chi-square test. At the leisure time, team sports were preferred by adolescents (33,3\%). Most of boys prefer soccer (44\%) and girls prefer to walk as a physical exercise $(35,7 \%)$. More than a half of leisure activities were characterized by sedentary type, however, active adolescents had more physical activities at the leisure time than sedentary adolescents $(\mathrm{p}=0,000)$.
\end{abstract}

Keywords: Motor Activity. Leisure Activities. Adolescent.

\section{Hábitos de la actividade física y recreacion para adolescentes}

\section{Resumen}

Este estudio examinó los hábitos de actividad física y de actividades recreativas en 216 adolescentes estudiantes de uno centro federal de educación tecnológica de Santa Catarina. El Questionário de Atividades Físicas Habituais y el Questionário de Autoavaliação do Estilo de Vida fueron utilizados. Los datos fueron tratados con estadística descriptiva e inferencial, se utilizaron la prueba " $t$ " de Student y Chi-cuadrado. Las actividades físico deportivas preferidas por los adolescentes en el tiempo libre son los deportes de equipo $(33,0 \%)$. Los chicos prefieren más al fútbol $(44 \%)$ y las niñas la caminata $(35,7 \%)$. Más de la mitad de las actividades recreati- 
vas $(56,8 \%)$ son sedentarias, pero los adolescentes activos eligen más actividades activas de lo que los sedentarios $(\mathrm{p}=0,000)$.

Palabras clave: Actividad Motora. Actividades Recreativas. Adolescente.

\section{Referências}

ALVES, J. G. B. et al. Prática de esportes durante a adolescência e atividade física de lazer na idade adulta. Revista Brasileira de Medicina do Esporte, v. 11, n. 5, set./out. 2005.

ANDRADE, A. Ocorrência e controle subjetivo do stress na percepção de bancários ativos e sedentários: a importância do sujeito na relação "atividade física e saúde". 2001. Tese (Doutorado em Engenharia de Produção) - Departamento de Engenharia de Produção e Sistemas, Universidade Federal de Santa Catarina, Florianópolis, 2001.

ATLANTIS, E.; et al. An effective exercise-based intervention for improving mental health and quality of life measures: a randomized controlled trial. Preventive Medicine, v. 39, p. 424-434, 2004.

BERGER, B. G.; et al. Relationships between expectancy of psychological benefits and mood alteration in joggers. International Journal of Sport Psychology, v. 29, p. 1-16, 1998.

BIDDLE, S. J. H.; et al. Increasing Demand for Sport and Physical Activity by Girls. Sportscotland. Disponível em: < www.sportscotland.org.uk>. Acesso em: 05 mai. 2006.

BIDDLE, S.; MUTRIE, N. Psychology of Physical Activity: determinants, well-being and interventions. London: Routledge, 2001.

CENTER OF DESEASE CONTROL (CDC). Physical activity and health: a report of the surgeon general. U.S. Department of Health and Human Services: Atlanta, 2006.

. Physical Activity Guidelines for Americans. U.S. Department of Health and Human Services: Atlanta, 2008.

CONTI, M. A., FRUTUOSO, M. F. P., GAMBARDELlA, A. M. D. Excesso de peso e insatisfação corporal em adolescentes. Rev. Nutr. Campinas, v. 18, n. 4, p. 491-497, 2005. 
DE-BOURDEAUDJUIJ, I.; et al. Stages of change for physical activity in community sample of adolescents. Health Education Research, v. 20, n. 3, p. 357-366, 2004.

ESCULCAS, C.; MOTA, J. Actividade física e práticas de lazer em adolescentes. Revista Portuguesa de Ciências do Desporto, v. 5, n. 1, p. 69-76, 2005.

GOMES, V. B.; SIQUEIA, K. S.; SICHIERI, R. Atividade Física em uma amostra probabilística da população do Município do Rio de Janeiro. Cadernos de Saúde Pública, Rio de Janeiro, v. 17, n. 4, jul./ago. 2001.

GRIESER, M. et al. Physical activity attitudes, preferences, and pratices in african american, hispanic, and caucasian girls. Healt Education e Behavior, v. 33, n. 1, p. 40-51, 2006.

GUEDES, D. P.; SANTOS, C. A.; LOPES, C. C. Estágios de mudança de comportamento e prática habitual de atividade física em universitários. Revista Brasileira de Cineantropometria e Desempenho Humano, v. 8, n. 4, p. 5-15, 2006.

LI, M.; DIBLEY, M. J.; SIBBRITT, D. W.; ZHOU, X.; YAN, H. Physical Activity and Sedentary Behavior in Adolescents in Xi'an City, China. Journal of Adolescent Health, v. 41, p. 99-101, 2007.

MASCARENHAS, L. P. G.; et al. Relação entre diferentes índices de atividade física e preditores de adiposidade em adolescentes de ambos os sexos. Revista Brasileira de Medicina do Esporte, v. 11, n. 4, jul./ago. 2005.

MATIAS, T. S.; et al. Estilo de vida, nível habitual de atividade física e percepção de autoeficácia de adolescentes. Rev. Ed. Física/UEM, Brasil. v. 20, n. 2, p. 235-243, 2009.

MATIAS, T. S.; et al. Satisfação corporal associada a prática de atividade física na adolescência. Motriz. Revista de Educação Física. UNESP, v. 16, n. 2, 2010.

MATSUDO, S.; MATSUDO, V.; ANDRADE, T. A. D.; OLIVEIRA, E. A. L.;BRAGGION, G. Nível de atividade física da população do estado de São Paulo: análise de acordo com o gênero, idade, nível só- 
cio-econômico, distribuição geográfica e de conhecimento. Revista Brasileira de Ciência e Movimento, v. 10, n. 4, p. 41-50, out. 2002.

NAHAS, M. V. Atividade física, saúde e qualidade de vida: conceitos e sugestões para um estilo de vida ativo. Londrina: Midiograf, 2001.

NOBRE, F. S. S. Hábitos de lazer, nível de atividade física e características somatomotoras de adolescentes. 2006. Dissertação (Mestrado em Ciências do Movimento Humano) - Departamento de Educação Física, Universidade do Estado de Santa Catarina, Florianópolis, 2006.

OEHLSCHLAEGER, M. H. K.; PINHEIRO, R. T.; HORTA, B.; GELATTI, C.; SAN'TANA, P. Prevalência e fatores associados ao sedentarismo em adolescentes de área urbana. Revista de Saúde Pública, v. 38, n. 2, p. 175-163, abr. 2004.

ORGANIZAÇÃO MUNDIAL DA SAÚDE. Physical activity and youth. Disponível em http://www.emro.who.int/whd2002/ReadingsSection2.htm. Acesso em: 20 jul. 2005.

PERKINS, D. F.; JACOBS, J. E.; BARBER, B. L.; ECCLES, J. S. Fitness activities during young adulthood childhood and adolescent sports participation as predictors of participation in sports and physical. Youth Society, v. 35, p. 495-520, 2004.

PETER S.; REGOLY-MEREI A.; BIRO L.; NAGY K.; ARATO G.; SZABO C.; VAMOS A.; ANTAL M. Lifestyle of Hungarian adolescents - Observations among metropolitan secondary school students. Annals of Nutrition and Metabolism, v. 52, n. 2, p.105-109, 2008.

PFEIFER, L. I. et al. A influência socioeconomica e de gênero no lazer de adolescentes. Psicologia: Teoria e Prática, v. 26, n. 3, p. 427-432, 2010.

PIRES, E. A. G.; et al. Hábitos de atividade física e o estresse em adolescentes de Florianópolis - SC, Brasil. Revista Brasileira Ciência e Movimento, Brasília, v. 12, n. 1, p.51-56, jul./mar. 2004.

REIS, R. S.; PETROSKI, E. L. Application of the social cognitive theory to predict stages of change in exercise for Brazilian adolescents. 
Revista Brasileira de Cineantropometria e Desempenho Humano, v. 2, n. 7, p. 62-68, 2005.

ROLIM , M. K. S. B. Auto-eficácia, estilo de vida e desempenho cognitivo de adolescentes ativos e sedentários. 2007. Dissertação (Mestrado em Ciências do Movimento Humano) - Departamento de Educação Física, Universidade do Estado de Santa Catarina, Florianópolis, 2007.

ROLIM, M. K. S. B.; et al. Estilo de vida de adolescentes ativos e sedentários. In: Fórum Internacional de Esportes, 6, 2007.

SALLES-COSTA, R.; et al. Gênero e prática de atividade física de lazer. Cadernos de Saúde Pública, v. 16, Supl, p. 325-333, 2003.

SANTOS, M.; et al. Variação sazonal na actividade física e nas práticas de lazer de adolescentes portugueses. Revista Portuguesa de Ciências do Desporto, v.5, n.2, p.192-201, maio 2005.

SARRIERA, J. C., TATIM, D. C., COELHO, R. P. S.; BÜSKER, J. Uso do Tempo Livre por Adolescentes de Classe Popular. Psicologia: Reflexão e Crítica, v. 20, n. 3, p. 361-367, 2007.

SEABRA, A. F. et al. Biological and socio-cultural determinants of physical activity in adolescents. Cadernos de Saúde Pública, v. 24, n. 4, p.721-736, 2008.

SILVA, R. C. R.; MALINA, M. R. Nível de atividade física em adolescentes do Município de Niterói. Cadernos de Saúde Pública, v. 16, n. 4, p. 1091-1097, out./dez. 2000.

SOUZA, G. S.; DUARTE, M. F. S. Estágios de mudança de comportamento relacionados à atividade física em adolescentes. Revista Brasileira de Medicina do Esporte, v. 11, n. 2, mar./abr. 2005.

TASSITANO, R. M.; et al. Atividade física em adolescentes brasileiros: uma revisão sistemática. Revista Brasileira de Cineantropometria e Desempenho Humano, v. 9, n. 1, p. 55-60, 2007.

TELAMA, R.; NUPPONEN, H.; PIÉRON, M. Physical activity among young people in the context of lifestyle. European Physical Education Review, v.11, n. 2, p.115-137, 2005. 
VASQUES D. G.; LOPES A. S. Fatores associados à atividade física e aos comportamentos sedentários em adolescentes. Rev. Bras. Cineantro. Des. Hum., 11(1):59-66, 2009.

VIANA, M. S.; ANDRADE, A.; MATIAS, T. S. Teoria da Autodeterminação: Aplicações no contexto da prática de exercícios físicos de adolescentes. Pensar a Prática, Goiânia, v. 13, n, 2, p. 1-18, 2010.

Recebido em: 18/07/2011

Revisado em: 27/01/2012

Aprovado em: 07/03/2012

\section{Endereço para correspondência}

camiliepacheco@yahoo.com.br

Camilie Pacheco Schmoelz

Universidade do Estado de Santa Catarina - UDESC

Av. Madre Benvenuta, 2007 - Itacorubi - Florianópolis - SC

CEP: 88.035-001 